\title{
Clinical presentation of extrahepatic portal vein obstruction: 10-year experience at a tertiary care hospital in Pakistan
}

\begin{abstract}
Farina M. Hanif, Ghous Bux Soomro, Sara Nazir Akhund, Nasir Hassan Luck, Syed Mudassir Laeeq, Zaigham Abbas, Syed Mujahid Hassan, Muhammed Mubarak ${ }^{1}$

Departments of Hepatogastroenterology and 'Pathology, Sindh Institute of Urology and Transplantation, Karachi, Pakistan
\end{abstract}

\section{ABSTRACT}

Objective: To evaluate the clinical presentation, possible etiological factors, management and outcome of patients in our hospital with extrahepatic portal vein obstruction (EHPVO). Materials and Methods: This study included patients with EHPVO followed up in our department during last 10 years. Patients of cirrhosis with EHPVO were excluded. Patients' clinical presentation, etiology of EHPVO, management and outcome results were analyzed. Results: Of 30 patients, $19(67.9 \%)$ were males. Median age was 12 years. Of 14 patients who underwent liver biopsy 9 had histological activity index stage of $1 / 6$. History of omphalitis and pulmonary tuberculosis was present in one case each. Of 22 patients with the available thrombophilia profile, nine patients had a deficiency of protein $\mathrm{C}$, five patients had a deficiency of protein $\mathrm{S}$, one each had reduced level $\mathrm{S}$ of anti-thrombin III and factor $\mathrm{V}$ mutation. The predominant presenting symptom was hematemesis (15 patients, 53.6\%). Seven patients (25\%) had splenomegaly. Three patients (10.7\%) had no esophageal varices on endoscopy. Three patients underwent splenectomy due to severe pancytopenia. Endoscopic retrograde cholangipancreatography was performed in four patients (14.3\%) due to portal biliopathy. Common bile duct stenting was performed in all four patients. Of them, one patient underwent splenorenal shunt operation for indication of hemobilia. One patient died at the age of 40 years, due to cholangitis and sepsis. Conclusions: Results from this study show that the anticoagulant deficiency is a common cause of EHPVO in our setup. Hematemesis is a common presenting symptom. Some of these patients have symptomatic portal biliopathy.

Key words: Extra hepatic portal vein obstruction, portal biliopathy, variceal bleed

Address for Correspondence: Dr. Farina Muhammed Hanif, Flat No. 101, Khanani Center, Block 3, Bahadurabad, Karachi, Pakistan. E-mail: farinahanif@hotmail.com



\section{INTRODUCTION}

The extrahepatic portal vein obstruction (EHPVO) is an important cause of portal hypertension in both children and adults. It may be caused by diverse etiological factors but in the majority of cases, the cause remains un-identified. Although the mortality due to variceal bleeding has been decreased due to effective endotherapy, significant morbidity is seen due to hypersplenism, growth retardation, impaired quality-oflife and portal biliopathy. ${ }^{[1]}$ There is scant information on the clinical presentation, etiology, management and outcome of patients with EHPVO in Pakistan.

We aimed to evaluate the clinical presentation, possible etiological factors, management and outcome of patients presenting to our hospital with EHPVO.

\section{MATERIALS AND METHODS}

This study is a retrospective case series of patients with portal hypertension diagnosed to have EHPVO and followed in Hepatogastroenterology Department during the period from January 2004 to August 2014. 
The hospital data were scrutinized for one of the following diagnosis: EHPVO, portal biliopathy, splenectomy, gastrointestinal bleed, and splenomegaly and shunt surgery. The patients of all age groups were included

Patients with portal vein obstruction and portal hypertension associated with chronic liver disease and patients without portal vein obstruction were excluded from the analysis.

Data items concerning patients' demographics, laboratory and radiological workup, endoscopic and surgical procedures, growth and development, were collected from the patients' clinical charts.

\section{Statistical analysis}

Patients' clinical presentation, etiology of EHPVO, management and outcome were analyzed. Statistical analysis was performed using SPSS version 19.0 (SPSS Inc., Chicago, IL, USA). All continuous variables were represented by mean \pm standard deviation and categorical variables by frequencies and percentages.

\section{RESULTS}

A total of 30 patients were included in the analysis, out of which $21(70 \%)$ were males while females were $9(30 \%)$. The age at presentation was $16.2 \pm 12.1$ years (median 12 years; range: $3-50$ years). Majority of patients were below 20 years $(80 \%)$. Only six patients were above 20 years. Table 1 shows the clinical characteristics of patients. A review of the growth chart (for weight) was carried out in 24 eligible patients ( $<20$ years of age). The growth charts were not available for eight patients. Of the remaining

\begin{tabular}{|c|c|}
\hline Patients clinical characteristics & Values \\
\hline Age, mean \pm SD & $16.2 \pm 12.1$ \\
\hline \multicolumn{2}{|l|}{ Age distribution, decade wise (\%) } \\
\hline$<10$-year & $12(40)$ \\
\hline $11-20$ years & $12(40)$ \\
\hline $21-30$ years & $1(3.3)$ \\
\hline $31-40$ years & $2(6.7)$ \\
\hline 41 years and above & $3(10)$ \\
\hline \multicolumn{2}{|l|}{ Gender, $n(\%)$} \\
\hline Male & $21(70)$ \\
\hline Female & $9(30)$ \\
\hline \multicolumn{2}{|c|}{ Clinical presentation and imaging findings } \\
\hline Hematemesis, $n(\%)$ & $15(50)$ \\
\hline Melena, $n(\%)$ & $2(6.7)$ \\
\hline Jaundice, $n(\%)$ & $3(9.9 \%)$ \\
\hline Splenomegaly, $n$ (\%) & $8(26.7)$ \\
\hline Liver size, $\mathrm{cm}$, mean $\pm \mathrm{SD}$ & $12.3 \pm 2.8$ \\
\hline Spleen size, $\mathrm{cm}$, mean \pm SD & $15.1 \pm 2.8$ \\
\hline
\end{tabular}

SD: Standard deviation
16 patients, $10(33.3 \%)$ were below the $25^{\text {th }}$ percentile while six showed normal growth development.

All patients had undergone ultrasound (US) of the abdomen (conventional and Doppler) for the confirmation of diagnosis of EHPVO. Computed tomography (CT) was performed in 15 patients while magnetic resonance imaging (MRI) portography was performed in three patients. In CT scan findings, along with cavernous transformation, portal vein thrombosis (PVT) was identified in three patients $(20 \%)$ while in remaining portal vein was not visualized. In magnetic resonance (MR) portography, all three patients had cavernous transformation.

The most common presenting symptoms were upper gastrointestinal bleed manifesting as hematemesis (15 patients, $50 \%$ ) while two patients $(6.7 \%)$ had melena only. In eight patients $(26.7 \%)$, splenomegaly was the only finding at presentation. Jaundice was presenting feature in three patients $(9.9 \%)$ while diarrhea was present in one patient $(3.3 \%)$.

Liver biopsy was performed in $16(53.3 \%)$ patients. There was no evidence of cirrhosis in any patient. Majority of patients had histological activity index stage of $1 / 6(30 \%)$.

One patient had a history of omphalitis, and another one had been treated for pulmonary tuberculosis in the past. Detailed prothrombotic profiles were available in 24 patients, including: Protein S, protein C, antithrombin III, factor V Leiden mutations. Eight patients had low levels of protein $\mathrm{C}$, three patients had a low level of protein $\mathrm{S}$, and 2 had reduced level of anti-thrombin III while one had a low level of factor $\mathrm{V}$ mutation. In remaining 13 patients, the levels were normal. In fifteen patients (50\%), no cause was identified. Table 2 shows the results of laboratory investigations of patients.

All patients have undergone upper gastrointestinal endoscopy. Three patients $(10 \%)$ had no esophageal varices. Esophageal banding was done in 23 patients $(76.7 \%$ ) while in remaining four patients sclerotherapy was performed. No complications of sclerotherapy or banding were reported. Nine patients $(30 \%)$ had bleeding the episode during follow-up. Along with esophageal varices ten patients $(33 \%)$ had gastric varices and it was dealt with $\mathrm{N}$-acetyl butyryl injection. Table 3 shows the details of endoscopic and surgical procedures done on these patients.

Endoscopic retrograde cholangipancreatography (ERCP) was performed in four patients $(12.1 \%)$. In two patients, it was done at the time of presentation while in additional two patients, it was performed during follow-up. Two patients had common bile duct (CBD) stone while all 


\begin{tabular}{|c|c|}
\hline Laboratory parameters & Values \\
\hline Hemoglobin (g/dl), mean \pm SD & $9.5 \pm 3.05$ \\
\hline $\mathrm{TLC}$, mean $\pm \mathrm{SD}$ & $5.2 \pm 3.7$ \\
\hline Platelet per $\mathrm{mm}^{3}$, mean $\pm \mathrm{SD}$ & $104 \pm 91.7$ \\
\hline $\mathrm{PT}$, mean $\pm \mathrm{SD}$ & $13.0 \pm 2.2$ \\
\hline $\mathrm{INR}$, mean $\pm \mathrm{SD}$ & $1.2 \pm 0.2$ \\
\hline Total bilirubin $(\mathrm{mg} / \mathrm{dl})$, mean $\pm \mathrm{SD}$ & $3.0 \pm 6.8$ \\
\hline $\mathrm{ALT}(\mathrm{U} / \mathrm{L})$, mean $\pm \mathrm{SD}$ & $62.1 \pm 139.3$ \\
\hline $\mathrm{AST}(\mathrm{U} / \mathrm{L})$, mean $\pm \mathrm{SD}$ & $65.3 \pm 115$ \\
\hline $\mathrm{GGT}(\mathrm{U} / \mathrm{L})$, mean $\pm \mathrm{SD}$ & $41.3 \pm 72.7$ \\
\hline Alkaline phosphate (U/L), mean \pm SD & $219.1 \pm 283.3$ \\
\hline \multicolumn{2}{|l|}{ Liver biopsy } \\
\hline \multicolumn{2}{|l|}{ Stage ${ }^{*}, n(\%)$} \\
\hline $0 / 6$ & $3(10)$ \\
\hline $1 / 6$ & $9(30)$ \\
\hline $2 / 6$ & $3(10)$ \\
\hline $3 / 6$ & $1(3.3)$ \\
\hline Not done & $14(46.7)$ \\
\hline \multicolumn{2}{|c|}{$\begin{array}{l}\text { *Ishak modification of the Knodell HAI staging system. TLC: Total } \\
\text { leukocyte count, PT: Prothrombin time, INR: International normalization } \\
\text { ratio, ALT: Alanine aminotransferase, AST: Aspartate aminotransferase, } \\
\text { GGT: Gamma glutamyl transferase, SD: Standard deviation, HAl: Histological } \\
\text { activity index }\end{array}$} \\
\hline
\end{tabular}

Table 3: Endoscopic and surgical procedures on patients with extrahepatic portal vein obstruction

\begin{tabular}{lc}
\hline Endoscopic and surgical procedures & Values \\
\hline Upper endoscopy, $n$ (\%) & \\
Esophageal varices, $n(\%)$ & $27(90)$ \\
Grade I & $2(6.7)$ \\
Grade II & $6(20)$ \\
Grade III & $10(33.3)$ \\
Grade IV & $9(30)$ \\
Gastric varices, $n(\%)$ & $10(33.3)$ \\
EVBL, $n(\%)$ & $23(76.7)$ \\
EST, $n(\%)$ & $4(23.3)$ \\
ERCP, $n$ & 4 \\
CBD stone, $n$ & 2 \\
CBD stricture, $n$ & 4 \\
\hline
\end{tabular}

EVBL: Endoscopic variceal band ligation, EST: Endoscopic sclerotherapy, CBD: Common bile duct, ERCP: Endoscopic retrograde cholangipancreatography

four had CBD stricture. CBD stenting was performed in all four patients.

In only one patient, spleno-renal shunt operation was performed for the indication of hemobilia. Three patients underwent splenectomy due to severe pancytopenia. One patient died, aged 40 years, due to cholangitis and sepsis secondary to portal biliopathy.

\section{DISCUSSION}

Extrahepatic portal vein obstruction is one of the common causes of noncirrhotic portal hypertension in developing countries. ${ }^{[1]}$ Although $30 \%$ of variceal bleeders have EHPVO, ${ }^{[2]}$ it does not account for patients' mortality. ${ }^{[3]}$ Therefore, management of EHPVO is not limited to treatment of varices but it also involves treatment of significant morbidities like growth failure, portal biliopathy, hypersplenism and its complications, etc.

There are various speculated etiologies of EHPVO including trauma, sepsis, umbilical vein catheterization, dehydration, myeloproliferative disorder, coagulation defects, congenital anomalies of portal vein, malignancy, and cirrrhosis. ${ }^{[2,3]}$

Hypercoagulable state due to deficiencies of protein C, protein $\mathrm{S}$, antithrombin III, and prothrombin or excess production of procoagulants due to factor $\mathrm{V}$ Leiden or gene mutations have been associated as predisposing factors of venous thrombosis in adults. ${ }^{[1,4]}$ While studies in children reported that anticoagulant deficiencies are common but not inherited. ${ }^{[4,5]}$ Even with extensive workup, it is postulated that in patients with EHPVO, about 70\% of cases remain idiopathic. ${ }^{[6]}$

In our study, in $50 \%$ of patients, no cause of PVT was identified, although Janus Kinase mutation was not performed due to un-availability of test, none of our patient had any evidence or history of trauma, umbilical vein catheterization or malignancy at the time of presentation.

Fourteen out of 24 patients were found to have anticoagulant deficiencies, in which factor $\mathrm{V}$ Leiden and prothrombin gene mutations were found in one and two patients, respectively, which was consistent with the study of Sharma et al. ${ }^{[4]}$ Anticoagulation analysis was not performed in their parents to determine the genetic origin as its role is not well-defined in pathogenesis of EHPVO. ${ }^{[3]}$

Keeping in view the debatable role of anticoagulation therapy in adults and no role in children, none of our patients was offered therapy. ${ }^{[3]}$

Although most of the patients with EHPVO present with variceal bleed, ${ }^{[3]}$ bleeding from nongastrointestinal sites has also been reported. ${ }^{[6]}$ Other presentations include hypersplenism, abdominal pain due to splenic infarcts and jaundice due to portal biliopathy. Hemoperitoneum, hemobilia, bowel ischemia, and mesenteric vein thrombosis are rarely observed.

In our study, patients mostly presented with hematemesis $(50 \%)$ and incidental splenomegaly was seen in eight patients $(26.7 \%)$. Jaundice was present in three patients, out of which 2 had portal biliopathy while one had sepsis. In our study, only one patient developed hemophilia during 
the course of his illness and had undergone splenorenal shunt for its management.

On clinical examination, patients with EHPVO usually have no stigmata of chronic liver disease. Ascites is present in $13-21 \%$ of cases $^{[6]}$ which is consistent with our study where ascites was present in four patients $(13.3 \%)$ at the time of presentation.

For the diagnosis of EHPVO, Doppler US is used as the first-line radiological investigation. CT abdomen or MR portography not only provide diagnosis but also delineate anatomical pathway for shunt surgery. In patients with EHPVO, esophageal varices are reported to be found in $80-90 \%$ of cases $^{[3]}$ and gastric varices in $31-44 \%$. As compared to cirrhotic cases, the esophageal varices are often larger. ${ }^{[6]}$ The results of our study are consistent with the above-mentioned findings. Esophageal varices were found in $90 \%$ of the study population, and the majority of patients (19 patients, 63.3\%) had varices of grade III and IV. Gastric varices were also present in $33 \%$ of patients.

Conventional management of EHPVO is focused on control of variceal bleed followed by secondary prophylaxis. However, the prognosis is better compared to patients with chronic liver disease due to better liver reserve. Although endoscopic sclerotherapy (EST) and endoscopic variceal band ligation (EVBL) have comparable efficacy in variceal eradication, ${ }^{[6]}$ the data on EVBL in children are sparse. ${ }^{[7]}$ Therefore, EST is considered a conventional modality in children for treatment of esophageal varices. ${ }^{[8]}$ In our study, due to available expertise, varices in children were dealt with EVBL and EST was performed in four patients and no postprocedure complications were observed.

Portal biliopathy refers to abnormalities in biliary ductal walls due to portal hypertension. ${ }^{[9]}$ Its frequency in adults is $80-100 \%$ while it is symptomatic in only $5-38 \%$ patients. ${ }^{[3,9]}$ Poddar et al. ${ }^{[10]}$ reported 13 cases of portal biliopathy in children with only one patient being symptomatic.

The suggested mechanisms of the high incidence of portal biliopathy in patients with EHPVO are prolonged continuous external compressions by portal collaterals, ischemic injury to biliary wall leading to stricture or both. ${ }^{[3,6]}$ In our study, cavernous transformation was present in all patients but only 2 presented with jaundice at the time of presentation while two patients developed jaundice later in the follow-up period.

Biliary changes due to portal biliopathy lead to various ominous consequences such as cholangitis, choledocholithiasis, and secondary biliary cirrhosis ${ }^{[11]}$ Even with vigorous management, $4-10 \%$ of patients die due to these complications. ${ }^{[11,12]}$ Likewise in our study, only one unfortunate patient died due to cholangitis secondary to portal biliopathy at the time of presentation.

Endoscopic retrograde cholangipancreatography is a useful modality for the diagnosis of portal biliopathy, but as the majority of patients are asymptomatic at the time of presentation, it is reserved for cases where intervention is needed. ${ }^{[9]}$ Depending on the requirement, patients can undergo endoscopic sphincterotomy, stent or nasobiliary placement, stone extraction, mechanical lithotripsy, or stricture dilatation. ${ }^{[1]}$ Although MR cholangiopancreatography coupled with portography allows visualization of the biliary tree similar to ERCP, but in some cases, it gives false diagnosis of obstruction. ${ }^{[9]}$ Of 30, four of our patients underwent ERCP, and all had stricture dilatation and stent placement, but only two of them had stone extraction.

Extrahepatic portal vein obstruction leads to growth retardation in children, and it has been reported to be about $51-54.5 \%{ }^{[13,14]}$ The postulated mechanisms include portal enteropathy induced malabsorption and/or impaired growth-factor synthesis from liver due to shunting of blood away from it.

In our study, only 10 patients $(33.3 \%)$ were below $25^{\text {th }}$ percentile for growth development. The low prevalence can possibly be due to the fact that in $8(26.7 \%)$ patients, growth chart was not available.

Shunt surgery is chiefly indicated when endotherapy fails to control variceal bleeding or in cases of delayed complications of portal biliopathy. Other indications include growth failure, massive splenomegaly, impaired quality-of-life and ahead of bilioenteric anastomosis. ${ }^{[6,11]}$ Although in the era of endoscopic management, emergency shunt surgery is infrequent, but in this study, one patient underwent splenorenal shunt surgery due to the development of hemobilia. In this case, hemostasis was temporarily achieved with covered self-expandable metallic stent deployed in the CBD.

Overall, the prognosis of patients with EHPVO after control of variceal bleeding is almost $100 \%$ for the long-term survival. ${ }^{[15]}$ Even, the mortality rate with uncontrolled variceal bleeding is $<5 \% .{ }^{[3]}$

\section{CONCLUSIONS}

Extrahepatic portal vein obstruction is the common cause of noncirrhotic portal hypertension. Due to effective endoscopic and surgical management, the mortality from variceal bleeding has diminished markedly. Early and 
accurate diagnosis and appropriate treatment are the key factors for the improved prognosis.

\section{REFERENCES}

1. Weiss B, Shteyer E, Vivante A, Berkowitz D, Reif S, Weizman Z, et al. Etiology and long-term outcome of extrahepatic portal vein obstruction in children. World J Gastroenterol 2010;16:4968-72.

2. Mandal D, Dattaray C, Sarkar R, Mandal S, Choudhary A, Maity TK. Is pregnancy safe with extrahepatic portal vein obstruction? An analysis. Singapore Med J 2012;53:676-80.

3. Poddar U, Borkar V. Management of extra hepatic portal venous obstruction (EHPVO): Current strategies. Trop Gastroenterol 2011;32:94-102.

4. Sharma S, Kumar SI, Poddar U, Yachha SK, Aggarwal R. Factor V Leiden and prothrombin gene G20210A mutations are uncommon in portal vein thrombosis in India. Indian J Gastroenterol 2006;25:236-9.

5. Dubuisson C, Boyer-Neumann C, Wolf M, Meyer D, Bernard O. Protein C, protein $S$ and antithrombin III in children with portal vein obstruction. J Hepatol 1997;27:132-5.

6. Khanna R, Sarin SK. Non-cirrhotic portal hypertension - Diagnosis and management. J Hepatol 2014;60:421-41.

7. Kim SJ, Kim KM. Recent trends in the endoscopic management of variceal bleeding in children. Pediatr Gastroenterol Hepatol Nutr 2013;16:1-9.
8. Poddar U, Thapa BR, Singh K. Endoscopic sclerotherapy in children Experience with 257 cases of extrahepatic portal venous obstruction. Gastrointest Endosc 2003;57:683-6.

9. Khan MR, Tariq J, Raza R, Effendi MS. Portal hypertensive biliopathy: Review of pathophysiology and management. Trop Gastroenterol 2012;33:173-8.

10. Poddar U, Thapa BR, Bhasin DK, Prasad A, Nagi B, Singh K. Endoscopic retrograde cholangiopancreatography in the management of pancreaticobiliary disorders in children. J Gastroenterol Hepatol 2001;16:927-31.

11. Dhiman RK, Behera A, Chawla YK, Dilawari JB, Suri S. Portal hypertensive biliopathy. Gut 2007;56:1001-8.

12. Chandra R, Kapoor D, Tharakan A, Chaudhary A, Sarin SK. Portal biliopathy. J Gastroenterol Hepatol 2001;16:1086-92.

13. Sarin SK, Bansal A, Sasan S, Nigam A. Portal-vein obstruction in children leads to growth retardation. Hepatology 1992;15:229-33.

14. Mehrotra RN, Bhatia V, Dabadghao P, Yachha SK. Extrahepatic portal vein obstruction in children: Anthropometry, growth hormone, and insulin-like growth factor I. J Pediatr Gastroenterol Nutr 1997;25:520-3.

15. Sarin SK, Agarwal SR. Extrahepatic portal vein obstruction. Semin Liver Dis 2002;22:43-58.

How to cite this article: Hanif FM, et al. Clinical presentation of extrahepatic portal vein obstruction: 10-year experience at a tertiary care hospital in Pakistan. J Transl Intern Med 2015;3:74-78

Source of Support: NIL, Conflict of Interest: NIL 